\title{
Improved Texture Enhanced Image Denoising
}

\author{
Jeetesh Kumar Rajak \\ M.Tech Student \\ Dept. of Electronics \& Communication \\ Mittal Institute of Technology Bhopal (MP)
}

\author{
Achint Chugh \\ Assistant Professor \\ Dept. of Electronics \& communication \\ Mittal Institute of Technology Bhopal (MP)
}

\begin{abstract}
here this work is introducing the new technique using the improved texture enhanced framework for image denoising. This technique is fast as compared to the higher order singular value decomposition (HOSVD) as we have in the previous work. The HOSVD technique simply compose in a cluster, alike Patches of noisy image in 3D heap, work out HOSVD factors of this heap, handles these factors by stiff thresholding, and turn upside down the HOSVD transmute to yield the final resultant image. Whereas improved texture enhanced image denoising have proven to be effective and robust in many image denoising tasks. It is experimentally demonstrating approximately 5 percent improved PSNR characteristics of ITEID technique on gray scale images. The ITEID process yields state-of-the-art outcomes on gray images, than HOSVD image data denoising process at moderately great noise stages.
\end{abstract}

\section{Keywords}

Image data denoising, singular value decomposition (SVD), HOSVD, patch Basis similarity, ITEID

\section{INTRODUCTION}

The image noise removable has a very prosperous history. An overabundance of distinct techniques has been offered, The image noise removable has a very prosperous history. An overabundance of distinct techniques has been offered, certain techniques we will analysis later. In present times, the transform-based methods mainly in combination through machine learning popularity have increased and achievements obtained on the basis of performance obtained. In this paper we have an uncomplicated graceful and useful algorithm that contributes to the impression of study a point wise shifting in term of transform corrupted image pixels with applying the nonlocal self-similarity of image. An effective explanation follows. Assumed an image $I_{n}$ which is the corrupted form of a basic clear image $I$, our objective is to improve a measurement of $I$ from $\mathrm{I}_{\mathrm{n}}$. We consider a zero mean i.i.d. Gaussian distribution of unchanged, common standard deviation $\sigma($ i. e, $\mathcal{N}(0, \sigma))$ like a noise model. The following steps are using in the noise removable algorithm: A heap consist of identical image patches is designed for all pixel and as a static spot size[15]. For each stack, higher order singular value decomposition (HOSVD) bases (3D for gray scale and 4D for color) are derived[15]. All heaps is planned on top of the bases and coefficients through values under a hard threshold are reduced to get a bunch of hypotheses [4]. The patches are reconstructed in image area and the bunch of hypotheses at all pixel averaged to achieve a noised free image [4]. The just free parameter is the patch size. This HOSVD based image noise removable algorithm get near to the advanced performance. We express example outcomes of our method on a gray scale depraved via noise from $\mathcal{N}(0,20)$ and a color version of the identical image in $\mathcal{N}(0,20)$ noise on $\mathrm{R}, \mathrm{G}$, and $\mathrm{B}$. Image noise generally occurs into the image transmission, quantization, acquisition and a many different processing steps. A digital image polluted through computer visualization tasks like classification, tracking, recognition, etc. and can affect several progressive image processing and noises leads to visible loss in image quality. The importance of image noise removable is therefore commonly accepted. Conventional image noise removable methods like as moving average filters, wavelet filter and Wiener filters banks are powerfully related to typical filtering[1].These filter created image noise removable methods are usually of low difficulty and can be simply achieved. But their performance is not all the time acceptable. By the improved computational capacity of modern processors, various advanced noise removable methods are now possible. With these method the Non-Local Means (NLM) technique [1], [2] has involved important awareness in current years. The Non-Local Means (NLM) remove the noise in an image pixel as the weighted sum of its corrupted neighbours, where every weight replicates the similarity among the local patch centred of the pixel to be noise removed and patch centred of the neighbour pixel. NLM familiarizes the noise removable process for every pixel and thus out performs conventional methods [1].

The proposed ITE noise removal method is a patch based method. Good priors of natural images are crucial to the success of an image denoising algorithm. A proper integration of different priors could further improve the denoising performance. In the proposed ITEID model, we adopt the following sparse nonlocal regularization term proposed in the non-locally centralized sparse representation (NCSR) model [14]. It should be noted that, for any gradient based image denoising model, we can easily adapt the proposed ITEID to it by simply modifying the Text Enhancement and adding an extra histogram specification operation. However, it is empirically found that our ITEID algorithm converges rapidly. The rest of the paper is termed as section 2 covers the HOSVD, sector 3 covers the proposed Improved Texture Enhanced Technique. Sector 4 with result analysis and sector 5 concludes the paper work.

\section{HIGHER ORDER SINGULAR VALUE DECOMPOSITIO [4]}

Generally in the NL-SVD procedure let consider original uncontaminated patch is $Q_{\text {ref }}$ and its reference patch $P_{\text {ref }}$ in the corrupted image. Now, suppose a situation where every $\mathrm{K}$ patches $\left\{\mathrm{P}_{\mathrm{i}}\right\}$ which is corrupted forms of $\mathrm{Q}_{\text {ref. }}$. In this situation, we notice that $\lim _{k \rightarrow \infty} \sum_{i=1}^{k} p_{i} p_{i}^{T}=Q_{r e f} Q_{r e f}^{T}+\sigma^{2}$ I. as a result for greater $\mathrm{K}$, we have an opportunity of being capable to approximate the singular value decomposition bases of $Q_{\text {ref }}$ and thus access the revelation estimator [4]. 
But, such a condition is not probable in most natural images, and the patches that succeed as alike will typically not be replica of $Q_{\text {ref }}$ modulo noise Therefore, we accept the following standard: If a bunch of patches are parallel to one another in the corrupted image, The noise removable should take this statement in the account and not noise removable them individually. With this is in mind, we cluster together identical patches and characterize them in the form of a 3D stack. The key point is that the cleaning is accomplished not only through the length and range of all singular (2D) patch, however similarly in the 3rd dimension so as to permit for similarity between intensity values at equivalent pixels of the dissimilar patches. The joint cleaning of several patches has been employed previous in the LJSCPW algorithm but with unchanging bases. On the other hand, in this work, we using this proposal to examine spatially adaptive bases. The advantage of our HOSVD methodology over NL-SVD, for de-noising a section of the boat image the application of coefficient thresholding for flattening of the structurally identical patches of size 64 by the NL-SVD and HOSVD transforms, respectively while the end two rows illustrate the cleaned patches after the averaging processes [15]. These figures disclose that HOSVD conserves the better textures on the tablecloth surface much finer than NL-SVD, which virtually removes those textures. We have also experimentally confirmed the importance of building the stack from similar patches: Randomly generated stacks create transforms that yield unclear and images..

\section{HOSVD FOR DENOISING}

We generate a stack of $\mathrm{K}-1$ parallel patches of given a $p \times p$ reference patch $P_{\text {ref }}$ in the corrupted image $I_{n}$, Here, likeness is demarcated and hence the significance of $\mathrm{K}$ diverges from one pixel to another pixel. Now we indicate the stack as $\mathrm{Z} \in \mathrm{R}^{\mathrm{p} \times \mathrm{p} \times \mathrm{k}}$. The HOSVD equation of given stack is,

$$
z=S \times_{1} U^{(1)} \times_{2} U^{(2)} \times_{3} U^{(3)}
$$

Where $U^{(1)} \in R^{p \times p}, U^{(1)} \in R^{p \times p}$, and $U^{(3)} \in R^{k \times k}$ are orthonormal matrices, and $\mathrm{S}$ is represent as $3 \mathrm{D}$ coefficient array of size $\mathrm{p} \times \mathrm{p} \times \mathrm{k}$ Here, the symbol $\times_{\mathrm{n}}$ given as nth mode tensor product well-defined in [12]. The orthonormal matrices $U^{(1)}, U^{(2)}$ and $U^{(3)}$ are, in repetition, calculated from the Singular Value Decomposition of the unfolding $Z_{(1)}, Z_{(2)}$ $\operatorname{andZ}_{(3)}$ respectively [12]. The exact Equation are the from,

$$
\begin{gathered}
Z_{(k)}=U^{(k)} \cdot S_{(k) .} \\
\left(U^{\bmod (k+1,3)} \otimes U^{\bmod (k+2,3)}\right)^{T}
\end{gathered}
$$

Where $1 \leq \mathrm{K} \leq 3$ (which are corresponding representations for the HOSVD). For $\mathrm{k} \times \mathrm{k}$ matrices is $\mathrm{O}(\mathrm{K} 3)$. For computational speed, we impose the constraint that $\mathrm{K} \leq 30$ The patches of $\mathrm{Z}$ are at that time estimated onto the HOSVD transform. The limitation for thresholding the transform coefficients are selected to be $\sigma \sqrt{2 \operatorname{logp}^{2} \mathrm{k}}$ again.

The stack of $\mathrm{Z}$ is at that time reassembled after inverting the transform [16], thereby filtering the every different patches into the collective and not take just reference patch. This gives extra flattening on every patches, which was essential because of the upper limit of $\mathrm{K} \leq 30$, dissimilar the case using NL-SVD,
We as well enhance the HOSVD denoising through a Wiener, let $\mathrm{Z}$ be a stack of parallel patches of the HOSVD cleaned image and $\mathrm{Zn}$ be the equivalent stack of the blaring image. Let the coefficients of $\mathrm{Z}$ and $\mathrm{Zn}$ on the HOSVD bases of $\mathrm{Z}$ be indicated as $\hat{c}$ and $c_{n}$, individually. Formerly, the cleaned

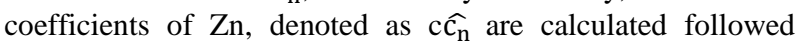
through the typical transform inversion and averaging:

$$
\widehat{c}_{\mathrm{n}}=\frac{\mathrm{c}_{\mathrm{n}} \hat{\mathrm{c}}^{2}}{\hat{\mathrm{c}}^{2}+\sigma^{2}}
$$

We call this second stage HOSVD.

\section{IMPROVED TEXTURE ENHANCED TECHNIQUE}

The noisy observation $\mathrm{y}$ of an unidentified unpolluted image $\mathrm{x}$ is usually modeled as

$$
\mathrm{y}=\mathrm{x}+\mathrm{v}
$$

where $\mathrm{v}$ is the additive white Gaussian noise (AWGN) with zero mean and standard deviation $\sigma$. The aim of image denoising is to estimate the wanted image $\mathrm{x}$ from $\mathrm{y}$. One popular method to image denoising is the variational method, in which the denoised image is found by

$$
\hat{x}=\arg \min _{x}\left\{\frac{1}{2 \sigma^{2}}\|y-x\|^{2}+\lambda \cdot R(x)\right\}
$$

Where $\mathrm{R}(\mathrm{x})$ represents some regularization word and $\lambda$ is a positive constant. The detailed form of $\mathrm{R}(\mathrm{x})$ depends on the employed image priors. One mutual problem of image noise removal methods is that the image well scale details such as texture structures will be over-smoothed. An over-smoothed image will have greatly frailer gradients than the original image. Naturally, a good estimation of $\mathrm{x}$ short of smoothing too much the textures should have a similar gradient distribution to that of $\mathrm{x}$. With this inspiration, we propose a gradient histogram preservation (GHP) model for texture enhanced image denoising

Assume that we have an approximation of the gradient histogram of $\mathrm{x}$, represent by $\mathrm{h}_{\mathrm{r}}$. In order to make the gradient histogram of without noised image $\hat{\mathrm{x}}$ nearly the same as the reference histogram $h_{r}$, we propose the following GHP based image denoising model:

$$
\begin{gathered}
\hat{x}=\operatorname{argmin}_{x, F}\left\{\frac{1}{2 \sigma^{2}}\|y-x\|^{2}+\lambda \cdot R(x)+\mu \| F(\nabla x)-\right. \\
\nabla x 2 \\
\text { s.t } h_{F}=h_{r}
\end{gathered}
$$

\section{DENOISING WITH GRADIENT HISTOGRAM PRESERVATION}

The proposed noise removal method is a patch based method. Let $\mathrm{x}_{\mathrm{i}}=\mathrm{R}_{\mathrm{i}} \mathrm{x}$ be a patch extracted at position $\mathrm{i}, \mathrm{i}=$ $1,2, \ldots, N$, where $R_{i}$ is the patch extraction operator and $N$ is the number of pixels in the image. Given a dictionary D, we sparselyencode the patch $\mathrm{x}_{\mathrm{i}}$ over $\mathrm{D}$, resulting in a sparse coding vector Once the coding vectors of all image patches are obtained,the whole image $\mathrm{x}$ can be recreated from

$$
x=D \circ \alpha=\left(\sum_{i=1}^{N} R_{i}^{T} R_{i}\right)^{-1} \sum_{i=1}^{N} R_{i}^{T} D \alpha_{i},
$$

where $\alpha$ is the concatenation of all $\alpha_{i}$.

Good priors of natural images are crucial to the success of an image denoising algorithm. A proper integration of different 
priors could further improve the denoising performance. For example, the methods in [9], [8], [10] integrate image local sparsity prior with nonlocal NSS prior and they have shown promising denoising results. In the proposed GHP model, we adopt the following sparse nonlocal regularization term proposed in the non-locally centralized sparse representation (NCSR) model [8]:

$$
R(x)=\sum_{i}\left\|\alpha_{i}-\beta_{i}\right\|_{1}, \quad s, t x=D \circ \alpha,
$$

where $\beta_{\mathrm{i}}$ is defined as the weighted average of $\alpha_{\mathrm{i}}^{\mathrm{q}}$ :

$$
\beta_{i}=\sum_{q} w_{i}^{q} \alpha_{i}^{q}
$$

: and $\alpha_{i}^{\mathrm{q}}$ the coding vector of the $\mathrm{q}^{\text {th }}$ nearest patch (denoted by $\mathrm{x})$ to $\mathrm{x}_{\mathrm{i}}$. The weight is defined $\operatorname{as} w_{i}^{q}=\frac{1}{w} \exp \left(-\frac{1}{h} \| \hat{x}_{i}-\right.$ xiq2 (' and xiqdenote the current estimates of xi and xiq, respectively), where $h$ is a predefined constant and $W$ is the normalization factor. More detailed explanations on NCSR can be found in .

By incorporating the above $\mathrm{R}(\mathrm{x})$ into Eq. (8), the proposed GHP model can be expressed as,

$$
\begin{gathered}
\hat{x}=\arg \min _{x, F}\left\{\frac{1}{2 \sigma^{2}}\|y-x\|^{2}+\lambda \cdot \sum_{i}\left\|\alpha_{i}-\beta_{i}\right\|_{1}+\right. \\
\mu F \nabla x-\nabla x 2 \\
\text { s.t } x=D \circ \alpha, h_{F}=h_{r}
\end{gathered}
$$

We use the method in [14] to construct the dictionary D adaptively. Based on the current estimation of image $\mathrm{x}$, we cluster its patches into $\mathrm{K}$ clusters, and for each cluster, a PCA dictionary is learned. Then for each given patch, we first check which cluster it belongs to, and then use the PCA dictionary of this cluster as D. Although in Eq. (8) the 11-norm regularization is imposed on $\left\|\alpha_{i}-\beta_{i}\right\|_{1}$ rather than $\left\|\alpha_{i}\right\|_{1}$, by introducing new variable $\vartheta_{i}=\alpha_{i}-\beta_{i}$, we can use the iterative shrinkage/thresholding method [11] to update $\vartheta_{\mathrm{i}}$ and then update $\alpha_{\mathrm{i}}=\vartheta_{\mathrm{i}}+\beta_{\mathrm{i}}$ This strategy is also used in [8]

To get the solution to the sub-problem in Eq. (8), we first use a gradient descent method to update $\mathrm{x}$ :

$x^{(k+1 / 2)}=x^{(k)}+\delta\left(\frac{1}{2 \sigma^{2}}\left(y-x^{(k)}\right)+\mu \nabla^{T}\left(g-\nabla x^{(k)}\right)\right)$

where $\delta$ is a pre-specified constant. Then, the coding coefficients $\alpha_{i}$ are updated by

$$
\alpha_{i}^{(k+1 / 2)}=D^{T} R_{i} x^{(k+1 / 2)}
$$

By using Eq. (6) to obtain $\beta_{\mathrm{i}}$, we further update $\alpha_{\mathrm{i}}$ by

$$
\alpha_{i}^{(k+1)}=S_{\lambda / d}\left(\alpha_{i}^{(k+1 / 2)}-\beta_{i}\right)+\beta_{i}
$$

where $\mathrm{S}_{-}=\mathrm{d}$ is the soft-thresholding operator, and $\mathrm{d}$ is a constant to guarantee the convexity of the surrogate function [11]. Finally, we update $\mathrm{x}^{(\mathrm{k}+1)}$ by

$$
x^{(k+1)}=D \circ \alpha^{(k+1)}=\left(\sum_{i=1}^{N} R_{i}^{T} R_{i}\right)^{-1} \sum_{i=1}^{N} R_{i}^{T} D \alpha_{i}^{(k+1)}
$$

Once the estimate of image $\mathrm{x}$ is given, we can update $\mathrm{F}$ by solving the following sub-problem:

$$
\min _{g, F}\|g-\nabla x\|^{2} \text { s.t. } h_{F}=h_{r}, g=F(\nabla x)
$$

Considering the equality constraint $\mathrm{g}=\mathrm{F}(\nabla \mathrm{x})$, we can substitute $g$ in $\|g-\nabla x\|^{2}$ with $F(\nabla x)$, and the sub-problem become

$$
\min _{F}\|F(\nabla x)-\nabla x\|^{2} \text { s.t. } h_{F}=h_{r},
$$

Noisy Image

Gradient Histogram Estimation

Graondient Histogram Preserving

Itrative Histogram Specification

Denosied Image

Fig. 1: Flowchart of the proposed texture enhanced image denoising framework.

To solve this sub-problem, by introducing $\mathrm{d}_{0}=$ $|\nabla x|$, thestandard histogram specification operator can be used to obtain the only feasible monotonic non-parametric transform $\mathrm{T}$ which makes the histogram of $\mathrm{T}(\mathrm{d} 0)$ the same as hr. Note that $(x-y)^{2} \leq((-x)-y)^{2}$

if the signs of $\mathrm{x}$ and $\mathrm{y}$ are the same. Since $\mathrm{F}(|\nabla \mathrm{x}|)=$ $\mathrm{T}(|\nabla \mathrm{x}|)$, to minimizing the squared error

$$
\|\mathrm{F}(\nabla \mathrm{x})-\nabla \mathrm{x}\| \text {, we should require that the sign of } \hat{\mathrm{F}}(\nabla \mathrm{x})
$$

is the same as that of $\nabla x$. Thus, we define $\hat{F}(\nabla x)$ as

$$
\hat{F}(\nabla x)=\operatorname{sgn}(\nabla x) T(|\nabla x|)
$$

Given $\hat{\mathrm{F}}(\nabla \mathrm{x})$, we then let $\mathrm{g}=\hat{\mathrm{F}}(\nabla \mathrm{x})$.

The proposed iterative histogram specification (IHS) based GHP algorithm is summarized in Algorithm 1. It should be noted that, for any gradient based image denoising model, we can easily adapt the proposed GHP to it by simply modifying the gradient term and adding an extra histogram specification operation

The GHP model in Eq. (10) is non-convex, and thus the proposed algorithm cannot be guaranteed to converge to a global optimum. However, it is empirically found that our GHP algorithm converges rapidly. Fig. shows an example convergence curve of the proposed GHP algorithm on image Bear (in Fig. 1). One can see that GHP converges within 15 20 iterations.

\section{REGION-BASED GHP}

The histogram constraint in Eq. (7) is global. If the image consists of different regions with different textures, GHP may generate some false textures in the less textured areas. To address this problem, we can partition the noisy image into several regions, estimate the reference gradient histogram of each region, and then apply GHP to each region for denoising As shown in Fig. 4, we suggest two schemes to partition the noisy image, resulting in two region-based GHP variants. The first scheme (Fig. 4(a)), namely S-GHP, is to employ k-means clustering method to roughly partition the image into $\mathrm{K}$ homogeneous

regions, while the second scheme (Fig. 4(b)), namely B-GHP, simply partitions the noisy image into, 


$$
K=\sqrt{K} \times \sqrt{K}
$$

blocks with equal size. Denote by $\left\{\Omega_{1}, \ldots, \Omega_{\mathrm{k}}, \ldots . \Omega_{\mathrm{k}}\right\}$ the partitioned regions. Each region $\Omega_{\mathrm{k}}$ has the corresponding reference gradient histogram $h_{r k}$, and we have a function $F_{k}$ to process the pixels within region $\Omega_{\mathrm{k}}$ :

$$
\min \sum(i, j) e \Omega_{k}\left(F_{k}\left((\Delta x)_{i j}\right)-(\Delta x)_{i j}\right)^{2} s, t h_{F}=h_{r k}
$$

We define an indicator function

$$
I_{\Omega_{k}}(i . j)=\left\{\begin{array}{c}
1, \text { if }(i, j) \in \Omega_{k} \\
0, \text { else }
\end{array}\right.
$$

The F $(\nabla \mathrm{x})$ for S-GHP/B-GHP can then be defined as

$$
F(\nabla x)=\sum_{k} F_{k}(\nabla x) I_{\Omega_{k}}
$$

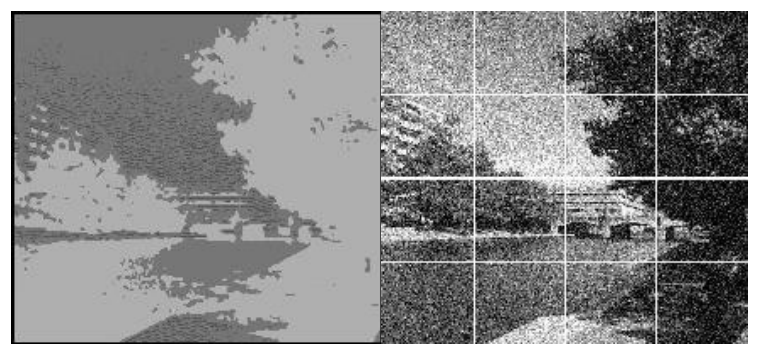

Fig. 2: Two image partition schemes. (a) The noisy image is partitioned into $K$ homogeneous regions by $k$-means clustering. (b) The noisy image is partitioned into $\sqrt{\mathbf{k}} \times \sqrt{\mathbf{k}}$ blocks

\section{REFERENCE GRADIENT HISTOGRAM ESTIMATION}

To apply the model in Eq. (7), we need to know the reference gradient histogram hr of original image $\mathrm{x}$. In this section, we propose a regularized deconvolution model to estimate the histogram $\mathrm{h}$ Assuming that the pixels in gradient image $\nabla \mathrm{x}$ are independent and distributed (i.i.d.), we can view them as the samples of a scalar variable, denoted by $x$. Then the normalized histogram of $\nabla \mathrm{x}$ can be regarded as a discrete approximation of the probability density function (PDF) of $\mathrm{x}$. For the AWGN v, we can readily model its elements as the samples of an i.i.d. variable, denoted by v. Since v $\mathrm{N}\left(0, \sigma^{2}\right)$ and let $\varepsilon=\nabla \mathrm{v}$ can then be well approximated by the i.i.d. Gaussian with PDF [38]

Since $y=x+v$, we have $\nabla y=\nabla x+\nabla v$. It is ready to model $\nabla y$ as an i.i.d. variable, denoted by $y$, and we have $y=x+\varepsilon "$. Let px be the PDF of $x$, and py be the PDF of $y$. Since $x$ and are independent, the joint $\operatorname{PDF} p(x, \varepsilon)$ is

$$
p(x, \varepsilon)=p_{x} \times p_{\varepsilon}
$$

Then the PDF $p_{y}$ is

$p_{y}=(y=t)=\int_{a} p_{x}(x=a) \times p_{\varepsilon}(\varepsilon=(t-a)) d a$.

If we use the normalized histogram $\mathrm{h}_{\mathrm{x}}$ and $\mathrm{h}_{\mathrm{y}}$ to approximate px and py, we can rewrite Eq. (21) in the discrete domain as,

$$
h_{y}=h_{x} \otimes h_{\varepsilon}
$$

where $\otimes$ denotes the convolution operator. Note that $h_{\varepsilon}$ can be obtained by discretizing $p_{\varepsilon}$, and $h_{y}$ can be computed directly from the noisy observation $y$.
Obviously, the estimation of $\mathrm{hx}$ can be generally modeled as a deconvolution problem

$$
h_{r}=\arg _{\operatorname{minh}}\left\{\left\|h_{y}=h_{x} \otimes h_{\varepsilon}\right\|^{2}+c \cdot R\left(h_{r}\right)\right\},
$$

where $c$ is a constant and $\mathrm{R}\left(\mathrm{h}_{\mathrm{x}}\right)$ is some regularization term based on the prior information of natural image's gradient histogram. We consider two kinds of constraints on hx. First, it has been shown that px (i.e., the continuous counter part of $\mathrm{h}_{\mathrm{x}}$ ) can be approximated by hyper-Laplacian distribution [13], [14], [15]. Considering that the real $h_{x}$ might deviate from the hyper-Laplacian distribution to some extent, we only require that hx should be close to the hyper-Laplacian distribution:

$$
p_{x} \approx C \cdot \exp \left(-k|x|^{y}\right)
$$

where $\mathrm{C}$ is the normalization factor,yand $\mathrm{k}$ are the two parameters of the hyper-Laplacian distribution. More specifically, we let $\mathrm{k} \in[0.001,3]$ and $\mathrm{y} \in[0.02,1.5]$. Second, each element of $h_{x}$ should be nonnegative. Based on these two constraints, gradient histogram estimation can be formulated as the following regularized de-convolution problem,

$h_{r}=$

$\arg \min _{h_{x}, C_{x, y}}\left\|h_{y}=h_{x} \otimes h_{\varepsilon}\right\|^{2}+\left\|h_{y}-C \cdot \exp \left(-k|x|^{y}\right)\right\|^{2}$

$$
\text { s.t. } h_{x} \geq 0
$$

which can be re-written as:

$$
\begin{gathered}
h_{r}=\operatorname{argmin}_{h_{x}, C_{x, y}}\left\{\begin{array}{c}
\left\|h_{y}=h_{x} \otimes h_{\varepsilon}\right\| \\
+\left\|h_{x}-C \cdot \exp \left(-k|x|^{y}\right)\right\|^{2} \\
+\eta\left\|h_{x}-h_{x}^{\prime}\right\|^{2}
\end{array}\right\} \\
\text { s.t. } \mathrm{h}_{\mathrm{x}}^{\prime} \geq 0
\end{gathered}
$$

We iteratively update $h_{x} h_{x}^{\prime}, C, \gamma$, and $k$ alternatively. Let $\mathrm{h}_{0}=\mathrm{C} \cdot \exp \left(-\mathrm{k}|\mathrm{x}|^{\mathrm{y}}\right), \mathrm{h}_{\mathrm{x}}$ is updated by

$$
h_{x}=\frac{\overline{F F T\left(h_{\varepsilon}\right)} \cdot F F T\left(h_{y}\right)+c F F T\left(h_{0}\right)+\eta F F T\left(h_{x}^{\prime}\right)}{F F T\left(h_{\varepsilon}\right) \cdot \overline{F F T\left(h_{\varepsilon}\right)}+c+\eta}
$$

here "•" denotes the element-wise multiplication, " : " denote $s$ the element-wise division, and " $*$ " denotes the complex conjugate operator. $\mathrm{h}_{\mathrm{x}}^{\prime}$ is updated by

$$
h_{x}^{\prime}(i)=\max \left(h_{x}(i), 0\right)
$$

C is updated by

$$
C=\frac{\sum_{i} \exp \left(-k|i|^{y}\right)}{\sum_{i} h_{x}(i)}
$$

$\gamma$ and $\mathrm{k}$ are updated based on gradient decent

$$
\begin{aligned}
& k^{(i+1)}=k^{(t)}+\tau \sum_{i} C|i|^{\gamma} \exp \left(-k^{(t)}|i|^{y}\right)\left(C \cdot \exp \left(-k^{(t)}|i|^{y}\right)-\right. \\
& h x(i), \\
& \gamma^{(t+1)}=\gamma^{(t)}+\rho \sum_{|i \neq 0|}\left\{\begin{array}{c}
C k^{(t)}|i|^{\gamma} \operatorname{In}|i| \exp \left(-k^{(t)}|i|^{y}\right) \\
\left(C \cdot \exp \left(-k^{(t)}|i|^{y}\right)-h_{x}(i)\right)
\end{array}\right\}
\end{aligned}
$$

\section{EXPERIMENTAL RESULTS}

All following simulations are done under the MATLAB r2010a environment with Intel Core CPU at $2.0 \mathrm{GHz}$. We display PSNR and SSIM values for denoising every image under independent $\mathrm{N}(0, \sigma)$ noise on all channel (where $\sigma \in$ $\{30,40,50\})$, in Tables 1 . Just as done for gray scale images, 
for all values, the noisy images were generated by adding Gaussian noise to the original image and converting the result to an image file ([0-255] range). We compare our results of proposed method with HOSVD we also implemented a Wiener filter step on top of HOSVD, which we term as HOSVD2.

Table 1. PSNR Results for Color Image (and SSIM Value on Gray scale Versions) Corrupted by $\mathbf{N}(0,40)$

\begin{tabular}{lrrc}
\hline S.no & HOSVD1 & HOSVD2 & ITEID \\
\hline $\mathbf{1}$ & $24.995,0.646$ & $25.327,0.671$ & $27.577,0.820$ \\
$\mathbf{2}$ & $28.278,0.711$ & $28.426,0.718$ & $28.800,0.844$ \\
$\mathbf{3}$ & $28.654,0.736$ & $28.900,0.745$ & $30.896,0.888$ \\
$\mathbf{4}$ & $29.654,0.803$ & $29.551,0.811$ & $32.305,0.909$ \\
\hline
\end{tabular}

For HOSVD, we calculate the patch similarity independently on the three channels gained later PCA with the distance threshold $\tau_{\mathrm{d}}=3 \sigma^{2} \mathrm{n}^{2}$ whereas in ITEID, One common problem of image denoising methods is that the image fine scale details such as texture structures will be over-smoothed

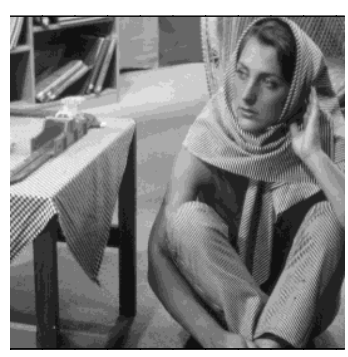

Original Image

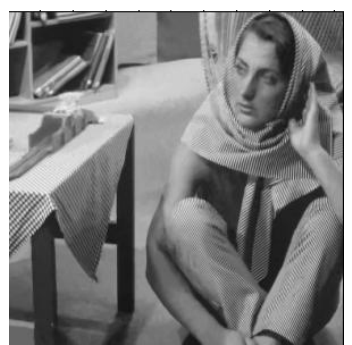

HOSVD1

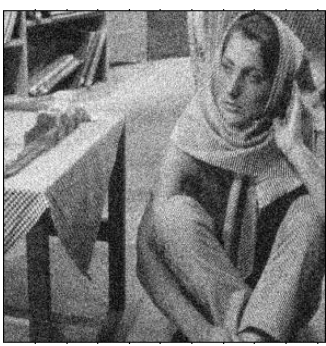

Noisy Image

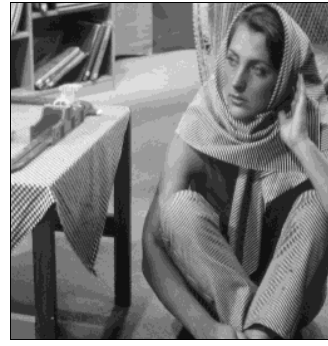

HOSVD2

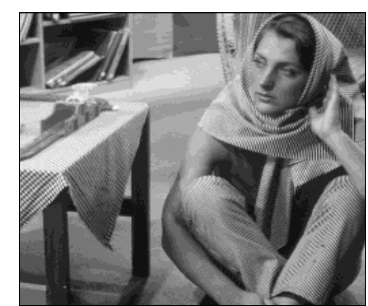

ITEID Image

Fig. 3. Left to right: Original image, noisy image (PSNR: 16.012) under of HOSVD (PSNR: 28.278), HOSVD2 (PSNR: 28.426), and denoised ITEID image (PSNR:32.305)
An over-smoothed image will have much weaker gradients than the original image. Intuitively, a good estimation of desired image without smoothing too much the textures should have a similar gradient distribution to that of desired image. An improved gradient histogram preservation (IGHP) model for texture enhanced image denoising provides good result. As compared to HOSVD/HOSVD2 method that uses the distance threshold $\tau_{\mathrm{d}}=3 \times 3 \sigma^{2} \mathrm{n}^{2}$. The patch-size used for all algorithms was $8 \times 8$.

\section{CONCLUSION}

The Improved texture enhanced image denoising (ITEID) methods clearly outperformed the NL Means, IHOSVD and IHOSVD2. Its PSNR values are slightly higher than those of IHOSVD and IHOSVD2; however, it out-performed IHOSVD and IHOSVD2 on some images. Thus, the denoising performance is then evaluated by computing its mean and standard deviation in terms of PSNR and SSIM .

\section{REFERENCES}

[1] Buades, B. Coll, and J. Morel, "A review of image denoising algorithms, with a new one," Multiscale Model. Simul., vol. 4, no. 2, pp. 490-530, 2005.

[2] Buades, B. Coll, and J.-M. Morel, "A non-local algorithm for image denoises," in IEEE Compu. Soc. Conf. Computer Vision and Pattern Recognition, Jun. 2005, vol. 2, pp. 60-65, vol. 2.

[3] L. de Lathauwer, "Signal Processing Based on Multilinear Algebra," PhD dissertation, Katholieke Universiteit Leuven, Belgium, 1997

[4] Ajit Rajwade, Anand Rangarajan and Arunava Banerjee, "Image Denoising Using the Higher Order Singular Value Decomposition" IEEE Transactions On Pattern Analysis and Machine Intelligence, Vol. 35, NO. 4, APRIL 2013.

[5] Yue Wu, Brian Tracey, Premkumar Natarajan, and Joseph P. Noonan James-Stein Type Center Pixel Weights for Non-Local Means Image Denoising IEEE Signal Processing Letters, Vol. 20, No. 4, April 2013.

[6] Wangmeng Zuo, Lei Zhang, Chunwei Song, David Zhang and Huijun Gao "Gradient Histogram Estimation and Preservation for Texture Enhanced Image Denoising"

[7] Jeetesh kumar Rajak, and Achint Chugh. "Review on Image Denoising by center pixel weights in Non-Local Means and elegant patch-based, machine learning method using higher order singular value decomposition "IJCA 2015.

[8] W. Dong, L. Zhang, G. Shi, and X. Li, "Nonlocally centralized sparse representation for image restoration," IEEE Trans. Image Process., vol.22, no. 4, pp. 16201630, Apr. 2013.

[9] J. Mairal, F. Bach, J. Ponce, G. Sapiro, and A. Zisserman, "Non-localsparse models for image restoration," in Proc. Int. Conf. Comput. Vis.,pp. 22722279, Sept. 29 2009-Oct. 22009.

[10] J. Jancsary, S. Nowozin, and C. Rother, "Loss-specific training of nonparametricimage restoration models: a new state of the art," in Proc.Eur. Conf. Comput. Vis., 2012. 
[11] I. Daubechies, M. Defriese, and C. DeMol, “An iterative thresholdingalgorithm for linear inverse problems with a sparsity constraint," Commun.Pure Appl. Math., vol. 57, no. 11, pp. 1413-1457, Nov. 2004.

[12] J. K. Patel and C. B. Read, "Handbook of the normal distribution," NewYork: Marcel Dekker, 1982.

[13] D. Krishnan, R. Fergus, "Fast image deconvolution using hyper-Laplacian priors," in Proc. Neural Inf. Process. Syst., pp. 1033-1041,2009.

[14] T. S. Cho, C. L. Zitnick, N. Joshi, S. B. Kang, R. Szeliski, and W. T.Freeman, "Image restoration by matching gradient distributions," IEEE.Trans. Pattern Anal. Mach. Intell., vol. 34, no. 4, pp. 683-694, Apr.2012.

[15] T. S. Cho, N. Joshi, C. L. Zitnick, S. B. Kang, R. Szeliski, and W. T.Freeman, "A content-aware image prior," in Proc. Int. Conf. Compu. Vis.Pattern Recognit., pp. 169-176, 13-18 June 2010.

[16] Donoho and I. Johnstone, "Ideal Spatial Adaptation by Wavelet Shrinkage," Biometrika, vol. 81, pp. 